FIAN/TD-01/99

ITEP/TH-14/99

\title{
Seiberg-Witten Curves and Integrable Systems t
}

\author{
A.Marshakov 2 \\ Theory Department, Lebedev Physics Institute, Moscow 117924, Russia \\ and \\ ITEP, Moscow 117259, Russia,
}

This talk gives an introduction into the subject of Seiberg-Witten curves and their relation to integrable systems. We discuss some motivations and origins of this relation and consider explicit construction of various families of Seiberg-Witten curves in terms of corresponding integrable models.

\section{Introduction}

Some time has been already passed after the observation of [1] that the effective theories for $\mathcal{N}=2$ vector supermultiplets [2, 3, 4] can be reformulated in terms of integrable systems. This connection, though not been clearly understood so far, has become already a beautiful example of appearance of hidden integrable structure in (multi-dimensional) quantum gauge theories and, thus, quite a popular topic at many different conferences. In this talk I was asked to review basic known facts of this relation and, in addition to brief explanation of some clear constituents of this correspondence, to present a list of problems which deserve further investigation.

The formulation of the Seiberg-Witten (SW) solution [2, 30 itself is very simple: the (Coulomb branch) low-energy effective action for the $4 \mathrm{D} \mathcal{N}=2$ SUSY Yang-Mills vector multiplets (supersymmetry requires the metric on moduli space of massless complex scalars from $\mathcal{N}=2$ vector supermultiplets to be of "special Kähler form" - or the Kähler potential $K(\mathbf{a}, \overline{\mathbf{a}})=\operatorname{Im} \sum_{i} \bar{a}_{i} \frac{\partial \mathcal{F}}{\partial a_{i}}$ should be expressed through a holomorphic function $\mathcal{F}=\mathcal{F}(\mathbf{a})$ - a prepotential) can be described in terms of auxiliary Riemann surface (complex curve) $\Sigma$ equipped with meromorphic 1-differential $d S$, possessing peculiar properties:

- The number of "live" moduli (of complex structure) of $\Sigma$ is strongly restricted (roughly "3 times" less than for generic Riemann surface). The genus of $\Sigma$ for the $S U(N)$ gauge theories is exactly equal 3 to the rank of gauge group - i.e. to the number of independent moduli.

- The variation of generating 1-form $d S$ over these moduli gives holomorphic differentials.

- The periods of generating 1-form

$$
\begin{gathered}
\mathbf{a}=\oint_{\mathbf{A}} d S \\
\mathbf{a}_{D}=\oint_{\mathbf{B}} d S
\end{gathered}
$$

give the set of "dual" masses - the W-bosons and the monopoles while the period matrix $T_{i j}(\Sigma)$ - the set of couplings in the low-energy effective theory. The prepotential is function of half of the variables (1),

\footnotetext{
${ }^{1}$ Talk given at the Edinburgh conference "Integrability: the Seiberg-Witten and Whitham Equations", 14-19 September 1998.

2e-mail address: mars@lpi.ac.ru, andrei@heron.itep.ru

${ }^{3}$ For generic gauge groups one should speak instead of genus - the dimension of Jacobian of a spectral curve - about the dimension of Prym variety. Practically it means that for other than $A_{N}$-type gauge theories one should consider the spectral curves with involution and only the invariant under the involution cycles possess physical meaning. We consider in detail only the $A_{N}$ theories, the generalization to the other gauge groups is straightforward: for example, instead of periodic Toda chains 5 , corresponding to $A_{N}$ theories 11, one has to consider the "generalized" Toda chains [6], first introduced for different Lie-algebraic series $(B, C, D, E, F$ and $G)$ in 7 .
} 
say $\mathcal{F}=\mathcal{F}(\mathbf{a})$, then

$$
\begin{gathered}
a_{D}^{i}=\frac{\partial \mathcal{F}}{\partial a_{i}} \\
T_{i j}=\frac{\partial a_{D}^{i}}{\partial a_{j}}=\frac{\partial^{2} \mathcal{F}}{\partial a_{i} \partial a_{j}}
\end{gathered}
$$

These data mean that the effective SW theory is formulated in terms of a classical finite-gap integrable system (see, for example, [12] and references therein) and their Whitham deformations [1]. The corresponding integrable models are well-known members of the $\mathrm{KP}$ /Toda family - for example, pure gauge theory corresponds to a periodic Toda chain [1, 6, the theories with broken $\mathcal{N}=4$ SUSY by the adjoint mass can be formulated in terms of the elliptic Calogero-Moser models [8], the theories with extra compact dimension (or Kaluza-Klein modes) give rise to appearance of relativistic integrable systems [9, 10, 11].

The aim of this talk is rather modest - to give some argumentation in favour of appearance of complex curves $\Sigma$ in the context of SUSY gauge theories and to present in clear way how the form of these curves can be explicitly found in by means of Lax representations of well-known finite-dimensional integrable systems. I should stress that the curves $\Sigma$ (and correspondent integrable systems) are auxiliary from physical point of view since the quantities (11), (2) effective theories needs depend only on moduli of SW curve or only on half of the variables of classical integrable system. This dependence is governed by integrable systems which are, in a sense, derivative from those we are going to consider below - the hierarchies of Whitham and (generalized) associativity equations. Both these subjects are, however, beyond the scope of these notes.

\section{Motivations and Origins}

In spite of absence at the moment any consistent and full explanations why the SW curves are identical to the curves of integrable systems, let us start from some physical motivations. We consider, first, the perturbative limit of $\mathcal{N}=2$ SUSY gauge theories and show that (degenerate) spectral curves and corresponding integrable systems appear already at this level. The situation is much more complicated for the non-perturbative picture and the only way to explain the origin of full SW curve exists in the framework of non-perturbative string theory or M-theory. In the second part of this section we shall discuss briefly how the Lax representations of the SW spectral curves arise in this context.

\subsection{Perturbative spectral curves}

Amazingly enough the relation between SW theories and integrable systems can be discussed already at the perturbative level, where $\mathcal{N}=2$ SUSY effective actions are completely defined by the 1-loop contributions (see [2] and references therein). The scalar field $\boldsymbol{\Phi}=\left\|\Phi_{i j}\right\|$ of $\mathcal{N}=2$ vector supermultiplet acquires nonzero VEV $\mathbf{\Phi}=\operatorname{diag}\left(\phi_{1}, \ldots, \phi_{N}\right)$ and the masses of "particles" $-W$-bosons and their superpartners are proportional to $\phi_{i j}=\phi_{i}-\phi_{j}$ due to the Higgs term $\left[A_{\mu}, \boldsymbol{\Phi}\right]_{i j}=A_{\mu}^{i j}\left(\phi_{i}-\phi_{j}\right)$ in the SUSY Yang-Mills action. These masses can be written altogether in terms of the generating polynomial

$$
w=P_{N}(\lambda)=\operatorname{det}(\lambda-\boldsymbol{\Phi})=\prod\left(\lambda-\phi_{i}\right)
$$

$(\boldsymbol{\Phi}$ - the adjoint complex scalar, $\operatorname{Tr} \boldsymbol{\Phi}=0$,$) via residue formula$

$$
m_{i j} \sim \oint_{C_{i j}} \lambda d \log w=\oint_{C_{i j}} \lambda d \log P_{N}(\lambda)
$$

which for a particular " $\infty$-like" contour $C_{i j}$ around the roots $\lambda=\phi_{i}$ and $\lambda=\phi_{j}$ gives rise to the Higgs masses. The contour integral (4) is defined on a complex plane - $\lambda$-plane with $N$ removed points: the roots of the polynomial (3) - a degenerate Riemann surface. The masses of monopoles are naively infinite in this limit, since the corresponding contours (dual to $C_{i j}$ ) start and end in the points where $d S$ obeys pole singularities. It means that the monopole masses, proportional to the squared inverse coupling, are renormalized in perturbation theory and defined naively up to the masses of particle states times some divergent constants.

The effective action (the prepotential) $\mathcal{F}$, or the set of effective charges $T_{i j}$ (2), are defined in $\mathcal{N}=2$ perturbation theory by 1-loop diagram giving rise to the logarithmic contribution

$$
\left(\delta^{2} \mathcal{F}\right)_{i j}=T_{i j} \sim \sum_{\text {masses }} \log \frac{(\text { mass })^{2}}{\Lambda^{2}}=\log \frac{\left(\phi_{i}-\phi_{j}\right)^{2}}{\Lambda^{2}}
$$


where $\Lambda \equiv \Lambda_{Q C D}$ and last equality is written only for pure gauge theories - since the only masses we have there are the Higgs masses (4). That is all one has in the perturbative weak-coupling limit of the SW construction, when the instanton contributions to the prepotential (being proportional to the degrees of $\Lambda^{2 N}$ (or $q^{2 N} \equiv e^{2 \pi i \tau N}$ - in the UV-finite theories with bare coupling $\tau$ ) are (exponentially) suppressed so that one keeps only the terms proportional to $\tau$ or $\log \Lambda$. We shall list several more examples below and demonstrate that these degenerated rational spectral curves can be already related to the family of trigonometric Ruijsenaars-Schneider and Calogero-Moser-Sutherland systems and the open Toda chain or Toda molecule.

Start with the original case of $S U(2)$ pure gauge theory. Eq. (3) turns into

$$
w=\lambda^{2}-u
$$

with $u=\frac{1}{2} \operatorname{Tr} \boldsymbol{\Phi}^{2}$. In the parameterization of [2 $X=w=e^{z}=\lambda^{2}-u, Y=w \lambda$ the same equation can be written as

$$
Y^{2}=X^{2}(X+u)
$$

and the masses (⿶) are now defined by the contour integrals of

$$
d S=\lambda d \log w=2 \frac{\lambda^{2} d \lambda}{\lambda^{2}-u}=\frac{\lambda d \lambda}{\lambda-\sqrt{u}}+\frac{\lambda d \lambda}{\lambda+\sqrt{u}}=\sqrt{X+u} \frac{d X}{X}
$$

One can easily notice that Eqs. (6), (7) and (8) can be interpreted as integration of the open $S L(2)$ (the Liouville) Toda chain with the co-ordinate $X=w=e^{q}$, momentum $p=\lambda$ and Hamiltonian (energy) $u$. The integration of generating differential $d S=p d q$ over the trajectories of the particles gives rise, in fact, to the monopole masses in the SW theory.

This is actually a general rule - the perturbative $\mathcal{N}=2$ theories of the "SW family" give rise to the "open" or trigonometric family of integrable systems - the open Toda chain, the trigonometric Calogero-Moser or Ruijsenaars-Schneider systems. This can be easily established at the level of spectrum (4) and the effective couplings (5) - the corresponding (rational) curves are (3) in the $N$-particle Toda chain case

$$
w=\frac{P_{N}^{(C M)}(\lambda)}{P_{N}^{(C M)}(\lambda+m)} \quad d S=\lambda \frac{d w}{w}
$$

for the trigonometric Calogero-Moser-Sutherland model and

$$
w=\frac{P_{N}^{(R S)}(\lambda)}{P_{N}^{(R S)}\left(\lambda e^{-2 i \epsilon}\right)} \quad d S=\log \lambda \frac{d w}{w}
$$

for the trigonometric Ruijsenaars-Schneider system. It is easy to see that (perturbative) spectra are given by general formula 11]

$$
\begin{gathered}
M=\phi_{i j} \oplus \frac{\pi n}{R} \oplus \frac{\epsilon+\pi n}{R} \\
N \in \mathbf{Z} .
\end{gathered}
$$

and contain in addition to the Higgs part $\phi_{i j}$ the Kaluza-Klein (KK) modes $\frac{\pi n}{R}$ and the KK modes for the fields with "shifted" by $\epsilon$ boundary conditions. The $\epsilon$ parameter can be treated as a Wilson loop of gauge field along the compact dimension - a kind of different (or dual) moduli in the theory and in a subclass of models it plays the role of the mass of the adjoint matter multiplet.

\subsection{Nonperturbative SW Curves, M-Theory and $\bar{\partial}$-equation}

As an example, let us consider, first, the case of pure $S U(2) \mathcal{N}=2$ gauge theory [2]. The gauge group has rank 1 and from the "integrable" point of view the situation is trivial, since the correspondent integrable model is "one-dimensional" (phase space has dimension two) and this case can be always solved explicitly. The full ("blown-up") spectral curve has the form

$$
w+\frac{\Lambda^{4}}{w} \underset{w=\bar{\Lambda}^{2} e^{z}}{=} 2 \Lambda^{2} \cosh z=\lambda^{2}-u \equiv P_{2}(\lambda)
$$

(cf. with (3)) and coincides with the equation relating the Hamiltonian (energy) $u$ with the co-ordinate $z=i q$ (or $z=q$ ) and momentum $\lambda=p$ of a particle moving into the $S L(2)$ Toda-chain potential - the well-known 
physical pendulum, instead of considered previously "Liouville wall" $t$. There are two other (hyper)elliptic parameterizations of the $S U(2) \mathrm{SW}$ curve: the first one was proposed in original paper [2]

$$
\begin{gathered}
Y^{2}=\left(X^{2}-4 \Lambda^{4}\right)(X+u) \\
X=P_{2}(\lambda)=\lambda^{2}-u \quad Y=\lambda y
\end{gathered}
$$

with $d S=(X+h) \frac{d X}{Y}, d t=\frac{d X}{Y}$ and another one used in 18]

$$
\begin{aligned}
& \tilde{y}^{2}=w^{3}+h w^{2}+\Lambda^{4} w \\
& w=\Lambda^{2} e^{z} \quad \tilde{y}=\lambda w
\end{aligned}
$$

endowed with $d S=\tilde{y} \frac{d w}{w}=\frac{\left(w^{2}+u w+1\right) d w}{w \tilde{y}}$ and $d t=\frac{d w}{\tilde{y}}$. Since any 1-dimensional system with conserving energy is integrable, (12) gives

$$
d t=\frac{d q}{p}=\frac{d z}{\lambda}=2 \frac{d \lambda}{y}
$$

where

$$
y^{2}=\left(\lambda^{2}-u\right)^{2}-4 \Lambda^{4}=4 \sinh ^{2} z
$$

so that

$$
t=\int \frac{d X}{Y}=2 \int \frac{d \lambda}{y}
$$

is the Abel map for (13) or (12). The (normalized) action integrals in two different ("sin- and sinh- Gordon") phases are the periods

$$
\left(a, a_{D}\right)=\oint_{(A, B)} d S=\oint_{(A, B)} p d q=\oint_{(A, B)} d q \sqrt{u+\Lambda^{2} \cos q}
$$

In the "sin-phase" if $u>>\Lambda^{2}$ the interaction is inessential and, the main effect for the integral (18) comes from

$$
a \sim \sqrt{u} \int_{A} d q \sim \sqrt{u} \times \text { const }
$$

In another "phase", corresponding to imaginary values of $q(q \rightarrow i q+\pi)$ the action integral (18) turns into

$$
a^{D}=\oint_{B} p d q=\oint_{B} d q \sqrt{u-\Lambda^{2} \cosh q}
$$

and in the first approximation is equal to

$$
a^{D} \sim \int_{B} d q \sqrt{u-\Lambda^{2} \cosh q} \sim \sqrt{u} \int_{B} d q \sim \sqrt{u} \times q_{*}=\sqrt{u} \log \frac{u}{\Lambda^{2}}
$$

where we by $q_{*}$ we have denoted the "turning point" $\Lambda^{2} e^{q_{*}}=u$. It is easy to see that the expressions (19) and (21) give the perturbative values of the W-boson $m_{W}^{2}=u$ and monopole $m_{M}^{2}=\left(\frac{m_{W}}{g_{Y M}^{2}}\right)^{2}$ masses in the $S U(2)$ SW theory. The prepotential for $u>>\Lambda^{2}$ is thus

$$
\mathcal{F}=\frac{u}{2}\left(\log \frac{u}{\Lambda^{2}}+o\left(\frac{\Lambda^{2}}{u}\right)\right)
$$

This is an obvious perturbative (1-loop) result of $\mathcal{N}=2$ SUSY Yang-Mills theory. The main hypothesis of [2] is that formulas (18) (and the prepotential or the set of coupling constants they define) are valid beyond the perturbation theory. In this case the r.h.s. of (22) would contain an infinite instanton series (in $\frac{\Lambda^{4}}{u^{2}}$ for the $S U(2)$ gauge group) but this can be encoded into a relatively simple modification of SW curves.

In the construction inspired by M-theory moduli arise either as positions of the D-branes $\phi_{i} \sim \frac{\left|r_{i}\right|}{\alpha^{\prime}}$ or as monodromies of the gauge fields $\epsilon=\oint A_{M} d x^{M}$ along the compactified directions (and are related to the positions of branes by T-duality), i.e. they are given by the set of data $\left(A_{M}, \boldsymbol{\Phi}^{(i)}\right)$. The perturbative spectral curves (6), (9), (10) correspond in this picture to the $p$-branes ( $p$-dimensional hypersurfaces with the $(p+1)$-dimensional

\footnotetext{
${ }^{4}$ Note, that for the SW theory one has to consider both phases - the sine-Gordon and the sinh-Gordon - of an integrable system together, that is why sometimes people speak in this respect about a complex integrable system.
} 
world volume) with the $\mathrm{D}(p-1)$-brane sources 15 . In particular case when the configuration can be described by two real (or one complex component) of both kind of fields $(\bar{A}, \boldsymbol{\Phi})$ one can define the full (smooth) spectral curves $\Sigma_{g}$ as a cover of some bare spectral curve $\Sigma_{0}$ - usually a torus, which can be naturally chosen when one has at least two compactified dimensions - by generalization of the equation (3)

$$
\operatorname{det}(\lambda-\boldsymbol{\Phi}(z))=0
$$

where $\boldsymbol{\Phi}=\boldsymbol{\Phi}(z)$ is now function (in fact 1-differential) on bare curve $\Sigma_{0}$ and obeys 113, 14]

$$
\bar{\partial} \Phi+[\bar{A}, \Phi]=\sum_{\alpha} J^{(\alpha)} \delta^{(2)}\left(P-P_{\alpha}\right)
$$

i.e. is holomorphic in the complex structure determined by $\bar{A}$. The invariants of $\bar{A}$ can be thought of as co-ordinates (one commuting set of variables) while the invariants of $\Phi$ as hamiltonians (another commuting set of variables) of an integrable system. It means that M-theory point of view implies that the VEV $\boldsymbol{\Phi}$ becomes a function on some base spectral curve $\Sigma_{0}$ - usually a cylinder or torus, appearing as a part of the (compactified) brane world volume and satisfies $\bar{\partial}$-equation. Such holomorphic (or, better, meromorphic) objects were introduced long ago [12] as Lax operators for the finite-dimensional integrable systems, holomorphically depending on some spectral parameter.

The (first-order) equation (24) arises from the BPS-like condition [16, 17 of the type $Q \psi=\Gamma_{M} D_{M} \Phi+$ $\Gamma_{M N} F_{M N}=0$ which determines the form of the Lax operator $\Phi$ and, thus, the shape of the curve (23). On torus with $p$ marked points $z_{1}, \ldots, z_{p}$ can be defined by $(i, j=1, \ldots, N)$

$$
\bar{\partial} \Phi_{i j}+\left(q_{i}-q_{j}\right) \Phi_{i j}=\sum_{\alpha=1}^{p} J_{i j}^{(\alpha)} \delta\left(z-z_{\alpha}\right)
$$

so that the solution has the form $\left(q_{i j} \equiv q_{i}-q_{j}\right)$ 局

$$
\begin{gathered}
\Phi_{i j}(z)=\delta_{i j}\left(p_{i}+\sum_{\alpha} J_{i i}^{(\alpha)} \partial \log \theta\left(z-z_{\alpha} \mid \tau\right)\right)+ \\
+\left(1-\delta_{i j}\right) e^{q_{i j}(z-\bar{z})} \sum_{\alpha} J_{i j}^{(\alpha)} \frac{\theta\left(z-z_{\alpha}+\frac{\operatorname{Im} \tau}{\pi} q_{i j}\right) \theta^{\prime}(0)}{\theta\left(z-z_{\alpha}\right) \theta\left(\frac{\operatorname{Im} \tau}{\pi} q_{i j}\right)}
\end{gathered}
$$

The exponential (nonholomorphic) part can be removed by a gauge transformation

$$
\Phi_{i j}(z) \rightarrow\left(U^{-1} \Phi U\right)_{i j}(z)
$$

with $U_{i j}=e^{q_{i} \bar{z}} \delta_{i j}$. The additional conditions to the matrices $J_{i j}^{(\alpha)}$

$$
\sum_{\alpha=1}^{p} J_{i i}^{(\alpha)}=0
$$

imply that the sum of all residues of a function $\Phi_{i i}$ vanishes, and

$$
\operatorname{Tr} J^{(\alpha)}=m_{\alpha}
$$

with $m_{\alpha}=$ const being some parameters ("masses") of a theory. The spectral curve equation becomes

$$
\mathcal{P}(\lambda ; z) \equiv \operatorname{det}_{N \times N}(\lambda-\mathbf{\Phi}(z))=\lambda^{N}+\sum_{k=1}^{N} \lambda^{N-k} f_{k}(z)=0
$$

where $f_{k}(z) \equiv f_{k}(x, y)$ are some functions (in general with $k$ poles) on the elliptic curve. If, however, $J^{(\alpha)}$ are restricted by

$$
\operatorname{rank} J^{(\alpha)} \leq l \quad l<N
$$

the functions $f_{k}(z)$ will have poles at $z_{1}, \ldots, z_{p}$ of the order not bigger than $l$. The generating differential, as usual, should be

$$
d S=\lambda d z
$$

\footnotetext{
${ }^{5}$ As usual, by $\theta_{*}(z \mid \tau) \equiv \theta_{11}(z \mid \tau)$ the (only on torus) odd $\left(\theta_{*}(0 \mid \tau)=0\right)$ theta-function is denoted.
} 
and its residues in the marked points $\left(z_{\alpha}, \lambda^{(i)}\left(z_{\alpha}\right)\right.$ ) (different $i$ correspond to the choice of different sheets of the covering surface) are related with the mass parameters (29) by

$$
m_{\alpha}=\operatorname{res}_{z_{\alpha}} \lambda d z \equiv \sum_{i=1}^{N} \operatorname{res}_{\pi_{(i)}^{-1} z_{\alpha}} \lambda^{(i)}(z) d z=\operatorname{res}_{z_{\alpha}} \operatorname{Tr} \boldsymbol{\Phi} d z
$$

We shall see that general form of the curve (30) coincides with the general curves arising in the SW theory (in many important cases torus should degenerate into a cylinder). For example, the $p=1, l=1$ case gives rise to the elliptic Calogero-Moser model (see eq. (56) below).

Thus, in the M-theory picture it becomes clear that full non-perturbative SW curves are smooth analogues of their degenerate perturbative cousins. This blowing up corresponds to the "massive" deformation of previous family of integrable systems, or, more strictly, the non-perturbative SW curves correspond to the family of periodic Toda chains 5 and Calogero-Moser-Ruijsenaars [25, 26, 27, 28] integrable models.

\section{Zoo of curves and integrable systems}

Now let us turn to the question of the zoo of SW's integrable systems - or some classification of relations between the SUSY YM theories and corresponding integrable models. We shall consider the cases of broken $\mathcal{N}=4$ SUSY theories, or $\mathcal{N}=2$ SUSY Yang-Mills with extra adjoint matter multiplets, theories with soft Kaluza-Klein (KK) modes or with extra 5th compact dimension and, as a separate question, theories with fundamental matter (all with the $S U(N)$ gauge group). The last issue is relatively less investigated yet, i.e. there still exist some open problems, mostly related with the "conformal" case $N_{f}=2 N$. Two first classes instead can be formulated in a unique way since there exists a "unifying" integrable system - the elliptic Ruijsenaars-Schneider model [27, 28] (with bare coupling $\tau$, "relativistic" parameter $R$ - the radius of compact dimension and an extra parameter $\epsilon$ - see (11)), giving rise to all known models of these two classes in its various degenerations [11]:

- If $R \rightarrow 0$ (with finite $\epsilon$ ) the mass spectrum (11) reduces to a single point $M=0$, i.e. all masses arise only due to the Higgs effect. This is the standard four dimensional $\mathcal{N}=2$ SUSY YM model associated with the periodic Toda chain. In this situation $\mathcal{N}=2$ SUSY in four dimensions is insufficient to ensure UV-finiteness, thus bare coupling diverges $\tau \rightarrow i \infty$, but the dimensional transmutation substitutes the dimensionless $\tau$ by the new dimensionful (and finite) parameter $\Lambda^{N}=e^{2 \pi i \tau}(\epsilon / R)^{N}$.

- If $R \rightarrow 0$ and $\epsilon \sim m R$ for finite $m$, then UV finiteness is preserved. The mass spectrum reduces to the two points $M=0$ and $M=m$. This is the four dimensional YM model with $\mathcal{N}=4$ SUSY softly broken to $\mathcal{N}=2$. The associated finite-dimensional integrable system [8] is the elliptic Calogero-Moser model [25, 26. The previous case is then obtained by Inosemtsev's 29] double scaling limit when $m \rightarrow 0$, $\tau \rightarrow i \infty$ and $\Lambda^{N}=m^{N} e^{2 \pi i \tau}$ is fixed.

- If $R \neq 0$ but $\epsilon \rightarrow i \infty$ the mass spectrum reduces to a single Kaluza-Klein tower, $M=\pi n / R, n \in Z$. This compactification of the five dimensional model has $N=1$ SUSY and is not UV-finite. Here $\tau \rightarrow i \infty$ and $\epsilon \rightarrow i \infty$, such that $2 \pi \tau-N \epsilon$ remains finite. The corresponding integrable system [9] is the relativistic Toda chain 30$]$.

- Finally, when $R \neq 0$ and $\epsilon$ and $\tau$ are both finite one distinguished case still remains: $\epsilon=\pi / 2$. 9 Here only periodic and anti periodic boundary conditions occur in the compact dimension. This is the case analyzed in 10, 11] and interested reader can find all details there.

\subsection{Toda chain}

This is the most well-known example of $S U(N)$ pure gauge theory 顿. The spectral curve is

$$
\begin{gathered}
w+\frac{\Lambda^{2 N}}{w}=P_{N}(\lambda) \\
P_{N}(\lambda) \equiv \operatorname{det}(\lambda-\mathbf{\Phi})=\lambda^{N}-\sum u_{k} \lambda^{k}
\end{gathered}
$$

\footnotetext{
${ }^{6}$ The case $\epsilon=0$ of fully unbroken five dimensional $N=2$ supersymmetry is of course also distinguished, but trivial: there is no evolution of effective couplings (renormalization group flows) and the integrable system is just that of $N$ non-interacting (free) particles.
} 
and the generating 1-form $d S=\lambda \frac{d w}{w}$ satisfies

$$
\left.\delta_{\text {moduli }} d S \equiv \delta_{\text {moduli }} d S\right|_{w=c o n s t}=\left(\delta_{\text {moduli }} \lambda\right) \frac{d w}{w}=\frac{\sum \lambda^{k} \delta u_{k}}{P_{N}^{\prime}(\lambda)} \frac{d w}{w}=\sum \frac{\lambda^{k} d \lambda}{y} \delta u_{k}=\text { holomorphic }
$$

where

$$
\begin{gathered}
y^{2}=\left(w-\frac{\Lambda^{2 N}}{w}\right)^{2}=P_{N}^{2}(\lambda)-4 \Lambda^{2 N} \\
\left.P_{N}^{\prime}(\lambda) \equiv \frac{\partial P_{N}}{\partial \lambda}\right|_{u=\text { const }}
\end{gathered}
$$

The spectral curve (34) corresponds to periodic $N$-particle problem in Toda chain [19, 20]. Let us demonstrate now how its form can be derived in the language of integrable systems using two different forms of the Lax representation with spectral parameter for periodic Toda chain.

The Toda chain system [5, 21] is a system of particles with only the neighbors pairwise exponential interaction with the equations of motion 7

$$
\frac{\partial q_{i}}{\partial t}=p_{i} \quad \frac{\partial p_{i}}{\partial t}=e^{q_{i+1}-q_{i}}-e^{q_{i}-q_{i-1}}
$$

where one assumes (for the periodic problem with the "period" $N$ ) that $q_{i+N}=q_{i}$ and $p_{i+N}=p_{i}$. It is an integrable system, with $N$ Poisson-commuting Hamiltonians, $h_{1}=\sum p_{i}=\mathrm{P}, h_{2}=\sum\left(\frac{1}{2} p_{i}^{2}+e^{q_{i}-q_{i-1}}\right)=E$, etc. Starting from naively infinite-dimensional system of particles $(37)$, the periodic problem can be formulated in terms of (the eigenvalues and the eigenfunctions of) two commuting operators: the Lax operator $\mathcal{L}$ (or the auxiliary linear problem for (37) 8)

$$
\lambda \Psi_{n}=\sum_{k} \mathcal{L}_{n k} \Psi_{k}=e^{\frac{1}{2}\left(q_{n+1}-q_{n}\right)} \Psi_{n+1}+p_{n} \Psi_{n}+e^{\frac{1}{2}\left(q_{n}-q_{n-1}\right)} \Psi_{n-1}
$$

and the second operator - in our case of periodic boundary conditions to be chosen as a monodromy or shift operator in a discrete variable - the number of a particle

$$
T q_{n}=q_{n+N} \quad T p_{n}=p_{n+N} \quad T \Psi_{n}=\Psi_{n+N}
$$

The existence of common spectrum of these two operators 9

$$
\mathcal{L} \Psi=\lambda \Psi \quad T \Psi=w \Psi \quad[\mathcal{L}, T]=0
$$

means that there is a relation between them $\mathcal{P}(\mathcal{L}, T)=0$ which can be strictly formulated in terms of spectral curve 22] $\Sigma: \mathcal{P}(\lambda, w)=0$. Here is an important difference with the perturbative case considered above, where only one (Lax) operator was really defined and there was no analog of the second $T$-operator. The generation function for the integrals of motion - the Toda chain Hamiltonians can be written in terms of $\mathcal{L}$ and $T$ operators and Toda chains possess two different (though, of course, equivalent) formulations of this kind.

In the first version the Lax operator (39) should be re-written in the basis of the $T$-operator eigenfunctions

\footnotetext{
${ }^{7}$ For simplicity in this section we consider a periodic Toda chain with coupling constant equal to unity. It is easy to restore it in all the equations; then it becomes clear that it should be identified with $\Lambda=\Lambda_{Q C D}$ - the scale parameter of pure $\mathcal{N}=2$ SUSY Yang-Mills theory.

${ }^{8}$ Equation (39) is the second-order difference equation and it has two independent solutions which we shall often denote below as $\Psi^{+}$and $\Psi^{-}$. In more general framework of the Toda lattice hierarchy these two solutions correspond to the two possible choices of sign in the time-dependent form of the Lax equation (39)$$
\pm \frac{\partial}{\partial t} \Psi_{n}^{ \pm}=\sum_{k} \mathcal{L}_{n k} \Psi_{k}^{ \pm}
$$

${ }^{9}$ Let us point out that we consider a periodic problem for the Toda chain when only the BA function can acquire a nontrivial factor under the action of the shift operator while the coordinates and momenta themselves are periodic. The quasiperiodicity of coordinates and momenta - when they acquire a nonzero shift - corresponds to the change of the coupling constant in the Toda chain Hamiltonians.
} 
and becomes the $N \times N$ matrix,

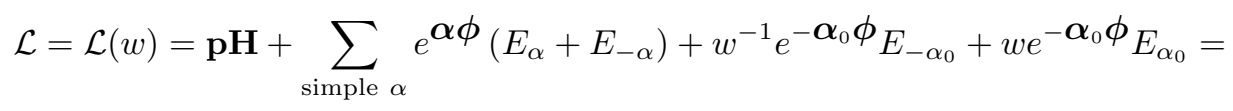

$$
\begin{aligned}
& =\left(\begin{array}{ccccc}
p_{1} & e^{\frac{1}{2}\left(q_{2}-q_{1}\right)} & 0 & & w e^{\frac{1}{2}\left(q_{1}-q_{N}\right)} \\
e^{\frac{1}{2}\left(q_{2}-q_{1}\right)} & p_{2} & e^{\frac{1}{2}\left(q_{3}-q_{2}\right)} & \ldots & 0 \\
0 & e^{\frac{1}{2}\left(q_{3}-q_{2}\right)} & p_{3} & 0 \\
\frac{1}{w} e^{\frac{1}{2}\left(q_{1}-q_{N}\right)} & 0 & 0 & & p_{N}
\end{array}\right)
\end{aligned}
$$

defined on a cylinder, i.e. it explicitly depends on spectral parameter $w$ - the eigenvalue of the shift operator (40). Matrix (42) is almost exactly three-diagonal, the only extra nonzero elements appear in the off-diagonal corners due to the periodic conditions (40) reducing in this way naively infinite-dimensional constant matrix (39) to a finite-dimensional one, but depending on spectral parameter $w$. The eigenvalues of the Lax operator (42) are defined from the spectral equation

$$
\mathcal{P}(\lambda, w)=\operatorname{det}_{N \times N}\left(\mathcal{L}^{T C}(w)-\lambda\right)=0
$$

Substituting the explicit expression (42) into (43), one gets:

$$
\mathcal{P}(\lambda, w)=w+\frac{1}{w}-P_{N}(\lambda)=0
$$

i.e. eq. 34, where $P_{N}(\lambda)$ is a polynomial of degree $N$, with the mutually Poisson-commuting coefficients:

$$
P_{N}(\lambda)=\lambda^{N}+h_{1} \lambda^{N-1}+\frac{1}{2}\left(h_{2}-h_{1}^{2}\right) \lambda^{N-2}+\ldots
$$

$\left(h_{k}=\sum_{i=1}^{N} p_{i}^{k}+\ldots\right)$, parameterizing (a subspace in the) moduli space of the complex structures of the hyperelliptic curves $\Sigma^{T C}$ of genus $N-1=\operatorname{rank} S U(N)$.

An alternative description of the same system arises when one (before imposing the periodic conditions!) solves explicitly the auxiliary linear problem (39) which is a second-order difference equation. To solve it one rewrites (39) as

$$
\Psi_{i+1}=\left(\lambda-p_{i}\right) \Psi_{i}-e^{q_{i}-\frac{q_{i+1}+q_{i-1}}{2}} \Psi_{i-1}
$$

or, since the space of solutions is 2-dimensional 10 , it can be rewritten as $\widetilde{\Psi}_{i+1}=L_{i}(\lambda) \widetilde{\Psi}_{i}$ where $\widetilde{\Psi}_{i}$ is a set of two-vectors and $L_{i}-$ a chain of $2 \times 2$ Lax matrices. After a simple "gauge" transformation $L_{i} \rightarrow U_{i+1} L_{i} U_{i}^{-1}$ where $U_{i}=\operatorname{diag}\left(e^{\frac{1}{2} q_{i}}, e^{-\frac{1}{2} q_{i-1}}\right)$ (and replacing $\left.p_{i} \rightarrow-p_{i}\right)$ these matrices can be written in the form [23]

$$
L_{i}(\lambda)=\left(\begin{array}{cc}
\lambda+p_{i} & e^{q_{i}} \\
e^{-q_{i}} & 0
\end{array}\right), \quad i=1, \ldots, N
$$

The matrices (47) obey quadratic r-matrix Poisson bracket relations: the canonical Poisson brackets of the co-ordinates and momenta of the Toda chain particles $\left\{q_{i}, p_{j}\right\}=\delta_{i j}$ can be equivalently rewritten in the "commutator" form

$$
\left\{L_{i}(\lambda) \stackrel{\otimes}{,} L_{j}\left(\lambda^{\prime}\right)\right\}=\delta_{i j}\left[r\left(\lambda-\lambda^{\prime}\right), L_{i}(\lambda) \otimes L_{j}\left(\lambda^{\prime}\right)\right]
$$

with the (i-independent!) numerical rational $r$-matrix $r(\lambda)=\frac{1}{\lambda} \sum_{a=1}^{3} \sigma_{a} \otimes \sigma^{a}$ satisfying the classical YangBaxter equation. As a consequence, the transfer matrix

$$
T_{N}(\lambda)=\prod_{N \geq i \geq 1}^{\curvearrowleft} L_{i}(\lambda)
$$

satisfies the same Poisson-bracket relation

$$
\left\{T_{N}(\lambda) \stackrel{\otimes}{,} T_{N}\left(\lambda^{\prime}\right)\right\}=\left[r\left(\lambda-\lambda^{\prime}\right), T_{N}(\lambda) \otimes T_{N}\left(\lambda^{\prime}\right)\right]
$$

\footnotetext{
${ }^{10}$ The initial condition for the recursion relation (46) consists of two arbitrary functions, say, $\Psi_{1}$ and $\Psi_{2}$, which are, of course, linear combinations of $\Psi^{+}$and $\Psi^{-}$from 38 .
} 
and the integrals of motion of the Toda chain are generated by another representation of spectral equation

$$
\begin{gathered}
\operatorname{det}_{2 \times 2}\left(T_{N}(\lambda)-w\right)=w^{2}-w \operatorname{Tr} T_{N}(\lambda)+\operatorname{det} T_{N}(\lambda)= \\
=w^{2}-w \operatorname{Tr} T_{N}(\lambda)+1=0
\end{gathered}
$$

(using that $\operatorname{det}_{2 \times 2} L(\lambda)=1$ leads to $\operatorname{det}_{2 \times 2} T_{N}(\lambda)=1$ ), or

$$
\mathcal{P}(\lambda, w)=w+\frac{1}{w}-\operatorname{Tr} T_{N}(\lambda)=w+\frac{1}{w}-P_{N}(\lambda)=0
$$

which coincides with (44). The polynomial $P_{N}(\lambda)=\operatorname{Tr} T_{N}(\lambda)$ in (52) is of degree $N$, its coefficients are the integrals of motion since

$$
\begin{gathered}
\left\{\operatorname{Tr} T_{N}(\lambda), \operatorname{Tr} T_{N}\left(\lambda^{\prime}\right)\right\}=\operatorname{Tr}\left\{T_{N}(\lambda) \stackrel{\otimes}{,} T_{N}\left(\lambda^{\prime}\right)\right\}= \\
=\operatorname{Tr}\left[r\left(\lambda-\lambda^{\prime}\right), T_{N}(\lambda) \otimes T_{N}\left(\lambda^{\prime}\right)\right]=0
\end{gathered}
$$

Finally, let us note that the Toda chain $N \times N$ Lax operator (42) by gauge transformation can be brought to another familiar form

$$
\begin{aligned}
& \mathcal{L}^{T C}(w) \rightarrow \tilde{\mathcal{L}}^{T C}=U^{-1} \mathcal{L}^{T C}(w) U=
\end{aligned}
$$

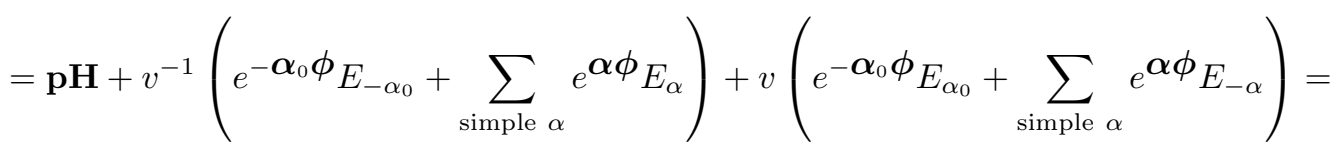

$$
\begin{aligned}
& =\left(\begin{array}{ccccc}
p_{1} & \frac{1}{v} e^{\frac{1}{2}\left(q_{2}-q_{1}\right)} & 0 & & v e^{\frac{1}{2}\left(q_{1}-q_{N}\right)} \\
v e^{\frac{1}{2}\left(q_{2}-q_{1}\right)} & p_{2} & \frac{1}{v} e^{\frac{1}{2}\left(q_{3}-q_{2}\right)} & \ldots & 0 \\
0 & v e^{\frac{1}{2}\left(q_{3}-q_{2}\right)} & p_{3} & & 0 \\
\frac{1}{v} e^{\frac{1}{2}\left(q_{1}-q_{N}\right)} & 0 & 0 & & p_{N}
\end{array}\right) \\
& U_{i j}=v^{i} \delta_{i j} \quad w \equiv v^{N}
\end{aligned}
$$

Formally this corresponds to change of gradation of the Toda chain Lax operator, the form (54) is especially natural relates the Toda chain Lax operator with the $\widehat{s l(N)}$ Kac-Moody algebra. In the form (54) the periodic Toda chain can be thought of as a special "double-scaling" limit of the $S L(N)$ Hitchin system on torus with a marked point - the Calogero-Moser model (see below). It is clear that the Lax operator (54) satisfies the $\bar{\partial}$-equation (24) on a cylinder with trivial gauge connection [24]

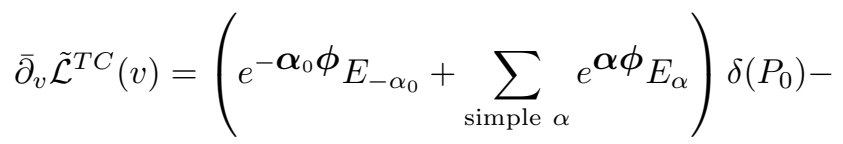

$$
\begin{aligned}
& -\left(e^{-\boldsymbol{\alpha}_{0} \phi_{E_{\alpha_{0}}}}+\sum_{\text {simple } \alpha} e^{\boldsymbol{\alpha} \boldsymbol{\phi}_{E_{-\alpha}}}\right) \delta\left(P_{\infty}\right)
\end{aligned}
$$

and they can be easily solved giving rise to (54).

\subsection{Broken $\mathrm{N}=4$ SUSY and the Elliptic Calogero-Moser Model}

Now, let us turn to the observation that the $N \times N$ matrix Lax operator 44) can be thought of as a "degenerate" case of the Lax operator for the $N$-particle Calogero-Moser system [26]

$$
\begin{gathered}
\mathcal{L}^{C M}(z)=\left(\mathbf{p H}+\sum_{\boldsymbol{\alpha}} F(\mathbf{q} \boldsymbol{\alpha} \mid z) E_{\boldsymbol{\alpha}}\right)= \\
=\left(\begin{array}{cccc}
p_{1} & F\left(q_{1}-q_{2} \mid z\right) & \ldots & F\left(q_{1}-q_{N} \mid z\right) \\
F\left(q_{2}-q_{1} \mid z\right) & p_{2} & \ldots & F\left(q_{2}-q_{N} \mid z\right) \\
& & \ldots & \\
F\left(q_{N}-q_{1} \mid z\right) & F\left(q_{N}-q_{2} \mid z\right) & \ldots & p_{N}
\end{array}\right)
\end{gathered}
$$


The matrix elements $F(q \mid z)=m \frac{\sigma(q+z)}{\sigma(q) \sigma(z)} e^{\zeta(q) z}$ are expressed in terms of the Weierstrass sigma-functions so that the Lax operator $\mathcal{L}(z)$ is defined on elliptic curve $E(\tau)$

$$
\begin{gathered}
y^{2}=\left(x-e_{1}\right)\left(x-e_{2}\right)\left(x-e_{3}\right) \\
x=\wp(z) \quad y=\frac{1}{2} \wp^{\prime}(z)
\end{gathered}
$$

or a complex torus with modulus $\tau$ and one marked point $z=0, x=\infty, y=\infty$. The Lax operator (566) corresponds to completely integrable system with Hamiltonians $h_{1}=\sum_{i} p_{i}=P, h_{2}=\sum_{i} p_{i}^{2}+m^{2} \sum_{i<j} \wp\left(q_{i}-\right.$ $q_{j}$ ), etc; the coupling constant $m$ in $4 \mathrm{D}$ interpretation plays the role of the mass of adjoint matter $\mathcal{N}=2$ hypermultiplet breaking $\mathcal{N}=4$ SUSY down to $\mathcal{N}=2[8]$.

¿From Lax representation (56) it follows that the spectral curve $\Sigma^{C M}$ for the $N$-particle Calogero-Moser system

$$
\operatorname{det}_{N \times N}\left(\mathcal{L}^{C M}(z)-\lambda\right)=0
$$

covers $N$ times elliptic curve (57) with canonical holomorphic 1-differential

$$
d z=\frac{d x}{2 y}
$$

The SW BPS masses $\mathbf{a}$ and $\mathbf{a}_{D}$ are now the periods of the generating 1-differential

$$
d S^{C M}=2 \lambda d z=\lambda \frac{d x}{y}
$$

along the non-contractable contours on $\Sigma^{C M} \Pi$.

In order to recover the Toda-chain system, one has to take the double-scaling limit [29], when $m$ and $-i \tau$ both go to infinity and

$$
q_{i}-q_{j} \rightarrow \frac{1}{2}\left[(i-j) \log m+\left(q_{i}-q_{j}\right)\right]
$$

so that the dimensionless coupling $\tau$ gets substituted by a dimensionful parameter $\Lambda^{N} \sim m^{N} e^{i \pi \tau}$. The idea is to separate the pairwise interacting particles far away from each other and to adjust the coupling constant simultaneously in such a way, that the interaction only of neighboring particles survives (and turns to be exponential). In this limit, the elliptic curve degenerates into a cylinder with coordinate $w=e^{z} e^{i \pi \tau}$ so that

$$
d S^{C M} \rightarrow d S^{T C}=\lambda \frac{d w}{w}
$$

The Lax operator of the Calogero system turns into that of the $N$-periodic Toda chain (42):

$$
\mathcal{L}^{C M}(z) d z \rightarrow \mathcal{L}^{T C}(w) \frac{d w}{w}
$$

and the spectral curve acquires the form (43). In the simplest example of $N=2$ the spectral curve $\Sigma^{C M}$ has genus 2. Indeed, in this particular case, Eq. (58) turns into

$$
\mathcal{P}(\lambda ; x, y)=\lambda^{2}-u+m^{2} x=0
$$

This equation says that with any value of $x$ one associates two points of $\Sigma^{C M}$

$$
\lambda= \pm \sqrt{u-m^{2} x}
$$

i.e. it describes $\Sigma^{C M}$ as a double covering of elliptic curve ramified at the points $x=\frac{u}{m^{2}}$ and $x=\infty$. In fact, $x=\frac{u}{m^{2}}$ corresponds to a pair of points on $E(\tau)$ distinguished by the sign of $y$. This would be true for $x=\infty$ as well, but $x=\infty$ is one of the branch points, thus, two cuts between $x=\frac{u}{m^{2}}$ and $x=\infty$ on every sheet of $E(\tau)$ touching at the common end at $x=\infty$ become effectively a single cut between $\left(\frac{u}{m^{2}},+\right)$ and $\left(\frac{u}{m^{2}},-\right)$. Therefore, we can consider the spectral curve $\Sigma^{C M}$ as two tori $E(\tau)$ glued along one cut, i.e. $\Sigma_{N=2}^{C M}$ has genus 2. It turns out to be a hyperelliptic curve (for $N=2$ only!) after substituting in (64) $x$ from the second equation to the first one.

\footnotetext{
${ }^{11}$ Let us point out that the curve $(58)$ has genus $g=N$ (while in general the genus of the curve defined by $N \times N$ matrix grows as $\left.N^{2}\right)$. However, integrable system is still $2(N-1)$-dimensional since the sum of the periods of (60) vanishes due to specific properties of $\lambda d z$ and there are only $N-1=g-1$ independent integrals of motion.
} 
Two holomorphic 1-differentials on $\Sigma^{C M}(g=N=2)$ can be chosen to be

$$
d v_{+}=d z=\frac{d x}{2 y}=\frac{\lambda d \lambda}{y} \quad d v_{-}=\frac{d z}{\lambda}=\frac{d x}{2 y \lambda}=\frac{d \lambda}{y}
$$

so that

$$
d S=2 \lambda d z=\lambda \frac{d x}{y}=\frac{d x}{y} \sqrt{u-m^{2} x}
$$

and

$$
\frac{\partial d S}{\partial u} \cong \frac{d x}{2 y \lambda}=d v_{-}
$$

The fact that only one of two holomorphic 1-differentials (66) appears at the r.h.s. of (68) is related to their different parity with respect to the $\mathbf{Z}_{2} \otimes \mathbf{Z}_{2}$ symmetry of $\Sigma^{C} M: y \rightarrow-y, \lambda \rightarrow-\lambda$ and $d v_{ \pm} \rightarrow \pm d v_{ \pm}$. Since $d S$ has certain (positive) parity, its integrals along the two of four elementary non-contractable cycles on $\Sigma^{C M}$ automatically vanish leaving only two non-vanishing quantities $a$ and $a_{D}$, as necessary for the $4 d$ interpretation. Moreover, two rest nonzero periods can be defined in terms of the "reduced" curve of genus $g=1$

$$
Y^{2}=(y \lambda)^{2}=\left(u-m^{2} x\right) \prod_{a=1}^{3}\left(x-e_{a}\right)
$$

equipped with $d S=\left(u-m^{2} x\right) \frac{d x}{Y}$ ㅍ. Since for this curve $x=\infty$ is no more a ramification point, $d S$ has simple poles when $x=\infty$ (on both sheets of $\Sigma_{\text {reduced }}^{C M}$ ) with the residues $\pm m$.

The opposite limit of the Calogero-Moser system with vanishing coupling constant $m^{2} \rightarrow 0$ corresponds to the $\mathcal{N}=4$ SUSY Yang-Mills theory with identically vanishing $\beta$-function. The corresponding integrable system is a collection of free particles and the generating differential $d S=\sqrt{u} \cdot d z$ is just a holomorphic differential on $E(\tau)$.

\subsection{Relativistic Toda Chain and the Ruijsenaars-Schneider Model}

So far we have considered the theories with only the Higgs and adjoint matter contribution to the spectrum (11). If, however, one adds also the soft KK modes [9], the resulting integrable systems would correspond to the Ruijsenaars-Schneider family [27, 28, 30], which is often called as the family of relativistic integrable models. The 1-loop contributions to the effective charge (5) are now of the form

$$
T_{i j} \sim \sum_{m} \log \left(a_{i j}+\frac{m}{R_{5}}\right) \sim \log \prod_{m}\left(R_{5} a_{i j}+m\right) \sim \log \sinh \left(R_{5} a_{i j}\right)
$$

i.e. coming from $4 \mathrm{D}$ up to $5 \mathrm{D}$ one should make a trigonometric substitution $a \rightarrow \sinh a R_{5}$, at least, in the formulas for the perturbative prepotential. The similar change of variables corresponds to relativization of integrable systems, which implies a sort of "Lie group generalization" of the "ordinary" integrable systems related rather to Lie algebras. The relativization of an integrable system replaces the momenta of particles by their exponentials and it results in the period matrices or effective charges of the (71) form. For example, in the case of $S U(2)$ pure gauge theory it gives instead of Hamiltonian of the Toda chain (12)

$$
\cosh z=\cosh p-\tilde{h}
$$

Generally, the net result is that in the case of relativistic Toda chain the spectral curve is a minor modification of (52)

$$
w+\frac{1}{w}=\left(\Lambda_{5} \lambda\right)^{-N / 2} P(\lambda),
$$

which can be again rewritten as a hyperelliptic curve in terms of the new variable $Y \equiv\left(\Lambda_{5} \lambda\right)^{N / 2}\left(w-\frac{1}{w}\right)$

$$
Y^{2}=P^{2}(\lambda)-4 \Lambda_{5}^{2 N} \lambda^{N}
$$

\footnotetext{
${ }^{12}$ This curve can be obtained by simple integration of the equations of motion since we again here deal with the only degree of freedom in our integrable system. The conserving energy $u=p^{2}+m^{2} \wp(q)$ gives

$$
t=\int \frac{d q}{\sqrt{h-m^{2} \wp(q)}}=\int \frac{d x}{\sqrt{\left(x-e_{1}\right)\left(x-e_{2}\right)\left(x-e_{3}\right)\left(u-m^{2} x\right)}}
$$

i.e. exactly the Abel map on the reduced curve (69)
} 
where $\lambda \equiv \mu^{2} \equiv e^{2 \xi}, \xi$ can be chosen as a spectral parameter of the relativistic Toda chain and $\Lambda_{5}$ is its coupling constant.

The most general picture in these terms corresponds to the "unifying" elliptic Ruijsenaars-Schneider model [9, 11. The Lax operator for the elliptic Ruijsenaars model is 28]

$$
\begin{gathered}
\mathcal{L}_{i j}^{R}=e^{P_{i}} \frac{\sigma\left(q_{i j}+z\right) \sigma(\epsilon)}{\sigma\left(q_{i j}+\epsilon\right) \sigma(z)} \\
e^{P_{i}}=e^{p_{i}} \prod_{k \neq i} \sqrt{\wp(\epsilon)-\wp\left(q_{i k}\right)},
\end{gathered}
$$

and in the trigonometric limit it turns into

$$
\begin{aligned}
\mathcal{L}_{i j}^{T R} & =e^{P_{i}} \frac{\sinh \left(q_{i j}+z\right) \sinh (\epsilon)}{\sinh \left(q_{i j}+\epsilon\right) \sinh (z)} \\
e^{P_{i}} & =e^{p_{i}} \prod_{k \neq i} \sqrt{1-\frac{\sinh ^{2} \epsilon}{\sinh ^{2}\left(q_{i k}\right)}}
\end{aligned}
$$

Introducing $\nu_{i}=e^{2 q_{i}}, \zeta=e^{2 z}$ and $q=e^{2 \epsilon}$ one finds that

$$
\begin{gathered}
\mathcal{L}_{i j}^{T R}=e^{P_{i}} \frac{\zeta \nu_{i}-\nu_{j}}{q \nu_{i}-\nu_{j}} \frac{1-q}{1-\zeta} \underset{\zeta \rightarrow \infty}{=}(q-1) \frac{e^{P_{i}} \nu_{i}}{q \nu_{i}-\nu_{j}}+\mathcal{O}\left(\frac{1}{\zeta}\right) \\
e^{P_{i}}=e^{p_{i}} \prod_{k \neq i} \frac{\sqrt{q \nu_{i}-\nu_{j}} \sqrt{\nu_{i}-q \nu_{j}}}{\nu_{i}-\nu_{j}}
\end{gathered}
$$

Only the leading term in (77) is often taken as an expression for the Lax operator of trigonometric Ruijsenaars system. The elliptic Ruijsenaars spectral curve acquires the form [28, 11]

$$
\operatorname{det}\left(\lambda-\mathcal{L}^{R}(z)\right)=\sum_{k=0}^{N}(-\lambda)^{N-k}\left\{\sum_{I_{k}} e^{P_{i_{1}}+\ldots+P_{i_{k}}} \tilde{D}_{I_{k}}\right\}=\sum_{k=0}^{N}(-\lambda)^{N-k} D_{k}(z \mid \epsilon) H_{k}=0
$$

where 13

$$
D_{k}(z \mid \epsilon)=(-)^{\frac{k(k-1)}{2}} \frac{\sigma^{k-1}(z-\epsilon) \sigma(z+(k-1) \epsilon)}{\sigma^{k}(z) \sigma^{k(k-1)}(\epsilon)}
$$

$\left(D_{0}(z \mid \epsilon)=1\right.$ and $\left.D_{1}(z \mid \epsilon)=1\right)$, and the generating differential is

$$
d S \cong \log \lambda d z
$$

In the simplest case of 1 degree of freedom $(N=2)$ Eq. (78) reads

$$
\lambda^{2}-u \lambda+D_{2}(z \mid \epsilon)=\lambda^{2}-u \lambda-\frac{\sigma(z-\epsilon) \sigma(z+\epsilon)}{\sigma^{2}(z) \sigma^{2}(\epsilon)}=\lambda^{2}-u \lambda+\wp(z)-\wp(\epsilon)=0
$$

and one gets a spectral curve identical to (64). The same sort of arguments as for the elliptic Calogero-Moser model shows that its full genus is $g=2$ while the $S L(2)$ integrable system lives effectively on reduced spectral curve of genus one. In trigonometric limit $(76),(77)$ the spectral curve $(78)$ turns into $(10)$. An interested reader can find the details concerning various limits of the Ruijsenaars-Schneider model in [11].

\subsection{Fundamental Matter}

Let us turn, finally, to the case of SUSY QCD - the SW theories with matter multiplets in the lowest dimensional representations of the gauge group. According to [3, 31] the spectral curves for $\mathcal{N}=2 \mathrm{SQCD}$ with gauge group

\footnotetext{
${ }^{13}$ In [11] we have used for technical reasons a slightly different form of the Ruijsenaars spectral curve, which can be obtained from Eq. (78) substituting $\lambda \rightarrow \lambda c(z)$ (or $D_{k}(z \mid \epsilon) \rightarrow D_{k}(z \mid \epsilon) c^{k}(z)$ ) with some (independent of Hamiltonians and $\epsilon$ ) function $c(z$ ). Such substitution does not change the "symplectic form" $\frac{d \lambda}{\lambda} \wedge d z$ and playing with such function some particular formulas can be brought to more simple form.
} 
$S U(N)$ and any number of matter multiplets $N_{f}<2 N$ have the form

$$
\begin{gathered}
y^{2}=P_{N}^{2}(\lambda)-4 \Lambda^{2 N-N_{f}} P_{N_{f}}(\lambda) \\
P_{N}(\lambda) \equiv P_{N}^{(0)}(\lambda)+R_{N-1}(\lambda) \equiv \prod_{i=1}^{N}\left(\lambda-\lambda_{i}(\boldsymbol{\Phi}, m)\right) \\
P_{N_{f}}(\lambda) \equiv \prod_{\alpha=1}^{N_{f}}\left(\lambda-m_{\alpha}\right)
\end{gathered}
$$

where $P_{N_{f}}(\lambda)$ and $R_{N-1}(\lambda)$ are "moduli independent" polynomials of $\lambda$ - i.e. they depend only on "external" masses of matter hypermultiplets [31], but spectral curves are still described by hyperelliptic equation. In contrast to pure gauge theory one runs immediately into a problem of parameter counting: $N-1$ moduli $\frac{1}{k} \operatorname{Tr} \boldsymbol{\Phi}^{k}$ together with $N_{f}$ masses $m_{\alpha}$ give $N+N_{f}-1$ and this is more than hyperelliptic moduli $2 g-2=2 N-4$ of the equation (82) for $N_{f}>N-3$. In other words, the moduli space of eq. (82) is too small to be parameterized by all parameters of the theory, that is why there are some complications in retranslating these models into the language of integrable systems [4]. However, the form (82) immediately implies [32] that for $\mathcal{N}=2$ SQCD there should exist a $2 \times 2$ representation of the kind (51) with monodromy matrix $T_{N}(\lambda)$, whose invariants have the form

$$
\begin{aligned}
\operatorname{Tr} T_{N}(\lambda) & =P_{N}(\lambda) \\
\operatorname{det} T_{N}(\lambda) & =P_{N_{f}}(\lambda)
\end{aligned}
$$

A natural proposal then is [35] to use a generalization of the $2 \times 2$ Toda chain Lax representation, i.e. to deform the Lax matrix (47) preserving the Poisson brackets (48) and (50). The monodromy matrix $T(\lambda)$ is again constructed by multiplication of $L_{i}(\lambda)$ 's (satisfying (48)) at different sites giving rise to the spectral curve equation

$$
\operatorname{det}\left(T_{N}(\lambda)-w\right)=0
$$

where from $T$-matrix one has to require (83). Wide class of such systems is given by the family of spin chains and to get most general form of the transfer matrix one should consider the inhomogeneous spin chain 15

$$
T_{N}(\lambda)=\prod_{N \geq i \geq 1}^{\curvearrowleft} L_{i}\left(\lambda-l_{i}\right)
$$

If Lax matrices $L_{i}(\lambda)$ were of the size $p \times p$ the equation (84) would have the form of $p$-th degree polynomial in $w$, if $p=2$ it is exactly the general case of (82) 16

$$
w+\frac{\operatorname{det} T_{N}(\lambda)}{w}=\operatorname{Tr} T_{N}(\lambda)
$$

or

$$
W+\frac{1}{W}=\frac{\operatorname{Tr} T_{N}(\lambda)}{\sqrt{\operatorname{det} T_{N}(\lambda)}} \equiv \frac{P_{N}(\lambda)}{\sqrt{P_{N_{f}}(\lambda)}}
$$

when $\operatorname{Tr} T_{N}(\lambda)$ has, like in the Toda chain case, degree $N$ but $\operatorname{det} T_{N}(\lambda)$ is not a unity, but some general polynomial of degree $N_{f} \leq 2 N$. The generating 1-form, according to general rules, becomes

$$
d S=\lambda \frac{d W}{W}
$$

The r.h.s. of the equations (86), (87) contain the dynamical variables of the spin system only in special combinations - the Hamiltonians (which are all in involution, i.e. mutually Poisson-commuting or the Casimir

\footnotetext{
${ }^{14}$ In particular, there are different ideas of interpretation gf the equation 82 (at least in the case of small $N_{f}<N$ ) in the context of integrable models, some of them can be found in [32, 33, 34]. We shall consider below, following [35, 37] only one of such options, which seems, however, to be the most attractive at present since it leads to a family of integrable systems which admits, for example, as in the case of adjoint matter, inclusion of the KK sector, or natural relativistic generalizations.

${ }^{15}$ For the Toda chain the inhomogenity parameters $l_{i}$ are not independent variables - they can be reabsorbed into the definition of momenta.

${ }^{16}$ We introduce new variable $W=\frac{w}{\sqrt{\operatorname{det} T_{N}(\lambda)}}$ in order to make analytic representation of spectral curve more symmetric. In the picture of M-theory these redefinitions are related with different brane pictures of the theories where presence of fundamental matter multiplets are caused by extra semi-infinite branes.
} 
functions and inhomogenities, commuting with all dynamical variables). The $2 \times 2$ Lax matrix for the simplest $\operatorname{sl}(2)$ rational $X X X$ chain is

$$
L(\lambda)=\lambda \cdot \mathbf{1}+\sum_{a=1}^{3} S_{a} \cdot \sigma^{a} .
$$

and the Poisson brackets on the space of the dynamical variables $S_{a}, a=1,2,3$ are implied by quadratic r-matrix relations (48) with the same rational $r$-matrix, as for the Toda chain [38].

The $r$-matrix relations (48) for the Lax matrix (89) are equivalent to well-known sl(2) commutation (Poisson bracket) relations

$$
\left\{S_{a}, S_{b}\right\}=i \epsilon_{a b c} S_{c},
$$

i.e. vector $\left\{S_{a}\right\}$ plays the role of angular momentum ("classical spin") variables giving the name "spin-chains" to the whole class of systems. The algebra (90) has an obvious Casimir operator (an invariant Poisson commuting with all generators $S_{a}$ ),

$$
K^{2}=\mathbf{S}^{2}=\sum_{a=1}^{3} S_{a} S_{a}
$$

so that

$$
\begin{gathered}
\operatorname{det}_{2 \times 2} L(\lambda)=\lambda^{2}-K^{2}, \\
\operatorname{det}_{2 \times 2} T_{N}(\lambda)=\prod_{1 \leq i \leq N} \operatorname{det}_{2 \times 2} L_{i}\left(\lambda-l_{i}\right)=\prod_{1 \leq i \leq N}\left(\left(\lambda-l_{i}\right)^{2}-K_{i}^{2}\right)= \\
=\prod_{1 \leq i \leq N}\left(\lambda+m_{i}^{+}\right)\left(\lambda+m_{i}^{-}\right)
\end{gathered}
$$

where we assumed that the values of spin $K$ can be different at different nodes of the chain, and 17

$$
m_{i}^{ \pm}=-l_{i} \mp K_{i} .
$$

Determinant of monodromy matrix (92) depends on dynamical variables only through the Casimirs $K_{i}$ of the Poisson algebra, and trace $P_{N}(\lambda)=\frac{1}{2} \operatorname{Tr}_{2 \times 2} T_{N}(\lambda)$ generates the Hamiltonians or integrals of motion. In the case of $s l(2) X X X$ spin chains, the spectral equation acquires exactly the form (86) or (87) where the number of matter multiplets, which determines the degree of polynomials in (83), $N_{f} \leq 2 N$ depends on a particular "degeneracy" of full chain.

In this picture the rational $\operatorname{sl}(2) X X X$ spin chain literally corresponds to a $N_{f}<2 N \mathcal{N}=2$ SUSY QCD. Things are not so simple with the "conformal" $N_{f}=2 N$ case when an additional dimensionless parameter appears - a naive toric generalization of the $X X X$-picture leads to the $X Y Z$ chain with the Hamiltonian structure given by elliptic Sklyanin algebra [38]. This model was proposed in [37] as a candidate for integrable system behind the $N_{f}=2 N$ theory, and, as it was discussed later (see, for example, [39] and references therein), it should be rather interpreted as a $4 \mathrm{D}$ theory with two extra compact dimensions (or a compactified 6D theory) 18. The condition $N_{f}=2 N$ which can be easily broken in $4 \mathrm{D}$ and $5 \mathrm{D}$ situations is very strict in $6 \mathrm{D}$ and one may think of the corresponding theory as of blowing up all possible compactified dimensions in the $N_{f}=2 N$ $4 \mathrm{D}$ theory with vanishing $\beta$-function.

In general, one finds, that the spectral curves for SUSY QCD with $N_{f}$ fundamental multiplets (and prepotentials) in $4 \mathrm{D}, 5 \mathrm{D}$ and $6 \mathrm{D}$ cases are described by similar formulas [39]. The spectral curves in all cases can be written in the form

$$
w+\frac{Q^{(d)}(\xi)}{w}=2 P^{(d)}(\xi)
$$

\footnotetext{
${ }^{17}$ Eq. (93) implies that the limit of vanishing masses, all $m_{i}^{ \pm}=0$, is associated with the homogeneous chain (all $\left.l_{i}=0\right)$ and vanishing spins at each site (all $\left.K_{i}=0\right)$.

${ }^{18}$ Indeed, in $6 \mathrm{D}$ theory with two extra compactified dimensions of radii $R_{5}$ and $R_{6}$ one should naively expect

$$
\begin{gathered}
T_{i j} \sim \sum_{m, n} \log \left(a_{i j}+\frac{m}{R_{5}}+\frac{n}{R_{6}}\right) \sim \\
\sim \log \prod_{m, n}\left(R_{5} a_{i j}+m+n \frac{R_{5}}{R_{6}}\right) \sim \log \theta\left(R_{5} a_{i j} \mid i \frac{R_{5}}{R_{6}}\right)
\end{gathered}
$$

i.e. coming from $4 \mathrm{D}$ or $5 \mathrm{D}$ to $D=6$ one should replace the rational (trigonometric) expressions by the elliptic functions, at least, in the formulas for the perturbative prepotential, the (imaginary part of) modular parameter being identified with the ratio of the compactification radii $R_{5} / R_{6}$.
} 


$$
W+\frac{1}{W}=\frac{2 P^{(d)}(\xi)}{\sqrt{Q^{(d)}(\xi)}}, \quad W=\frac{w}{\sqrt{Q^{(d)}(\xi)}}
$$

the generating differentials are

$$
d S=\xi d \log W
$$

and the perturbative part of the prepotential is always of the form

$$
\mathcal{F}=\frac{1}{4} \sum_{i, j} f^{(d)}\left(a_{i j}\right)-\frac{1}{4} \sum_{i, \alpha} f^{(d)}\left(a_{i}-m_{\alpha}\right)+\frac{1}{16} \sum_{\alpha, \beta} f^{(d)}\left(m_{\alpha}-m_{\beta}\right)
$$

The functions introduced $f^{(d)}$ are:

$$
\begin{gathered}
Q^{(4)}(\xi) \sim \prod_{\alpha}^{N_{f}}\left(\xi-m_{\alpha}\right), \quad Q^{(5)}(\xi) \sim \prod_{\alpha}^{N_{f}} \sinh \left(\xi-m_{\alpha}\right) \\
Q^{(6)}(\xi) \sim \prod_{\alpha}^{N_{f}} \frac{\theta_{*}\left(\xi-m_{\alpha}\right)}{\theta_{*}^{2}\left(\xi-\xi_{i}\right)} \\
P^{(4)} \sim \prod_{i}^{N}\left(\xi-a_{i}\right), \quad P^{(5)} \sim \prod_{i}^{N} \sinh \left(\xi-a_{i}\right) \\
P^{(6)} \sim \prod_{i}^{N} \frac{\theta_{*}\left(\xi-a_{i}\right)}{\theta_{*}\left(\xi-\xi_{i}\right)}
\end{gathered}
$$

(in $P^{(5)}(\xi)$, there is also some exponential of $\xi$ unless $N_{f}=2 N$ )

$$
\begin{gathered}
f^{(4)}(x)=x^{2} \log x \\
f^{(5)}(x)=\sum_{n} f^{(4)}\left(x+\frac{n}{R_{5}}\right)=\frac{1}{3}\left|x^{3}\right|-\frac{1}{2} \operatorname{Li}_{3}\left(e^{-2|x|}\right) \\
f^{(6)}(x)=\sum_{m, n} f^{(4)}\left(x+\frac{n}{R_{5}}+\frac{m}{R_{6}}\right)=\sum_{n} f^{(5)}\left(x+n \frac{R_{5}}{R_{6}}\right)= \\
=\left(\frac{1}{3}\left|x^{3}\right|-\frac{1}{2} \operatorname{Li}_{3, q}\left(e^{-2|x|}\right)+\text { quadratic terms }\right)
\end{gathered}
$$

so that

$$
f^{(4)^{\prime \prime}}=\log x \quad f^{(5)^{\prime \prime}}(x)=\log \sinh x \quad f^{(6)^{\prime \prime}}(x)=\log \theta_{*}(x)
$$

Note that, in $6 \mathrm{D}$ case, we have always $N_{f}=2 N$. The variables $\xi_{i}$ above are inhomogenities in integrable system, and, in $D=5,6$, there is a restriction $\sum a_{i}=\sum \xi_{i}=\frac{1}{2} \sum m_{\alpha}$ which implies that gauge moduli would be rather associated with $a_{i}$ shifted by the constant $\frac{1}{2 N} \sum m_{\alpha}$.

\section{Conclusion}

In this talk I have tried to formulate main issues of the correspondence between the Seiberg-Witten curves governing the exact solutions to $\mathcal{N}=2$ SUSY gauge theories and integrable systems. During last 4 years there was a lot of progress in this direction. However, there is still a lot of questions which are not yet understood and deserve further investigation. Let me, finally, point out at least some of them:

- The first, and the most essential question is still open: how to derive the SW - integrable systems correspondence from first principles. There is no clear answer to the question what means the dynamics governed by an integrable system directly in terms of SUSY gauge theory.

- The point which seems to be already clear is that SW construction itself becomes much more transparent from the point of view of non-perturbative string theory or M-theory. However, it is still many open questions in this direction - for example the M-theory picture of the Ruijsenaars-Schneider model, doubleelliptic systems etc. It is clear that the Ruijsenaars Lax operator (75) satisfies similar to (24) $\bar{\partial}$-equation, but there is no clear interpretation of this equation in terms of D-brane constructions. 
- I have already mentioned some general problems with fundamental matter. Of particular interest is the conformal $N_{f}=2 N$ case, which should be clearly related, on one hand, with double-elliptic systems and, on the other hand, with the K3 and elliptic Calabi-Yau compactifications of string and M-theory.

- In this talk only the $S U(N)$ gauge theories were considered in detail. I did not touch at all many problems related to the generalizations to the other gauge groups and representations. From the point of view of integrable systems this is a question, in part, about different representations of the Lax pairs for integrable systems and it is overlapped, thus, with the problem of different Lax pairs for the Calogero-Moser systems found recently in [40] and 41.

\section{Acknowledgements}

I am grateful to H.Braden, I.Krichever, A.Mironov and A.Morozov for illuminating discussions and to the organizers of the conference for nice time in Edinburgh. The work was partially supported by the RFBR grant 98-01-00344 and the INTAS grant 96-482.

\section{References}

[1] A.Gorsky, I.Krichever, A.Marshakov, A.Mironov and A.Morozov, Phys.Lett., B355 (1995) 466, hepth/9505035.

[2] N.Seiberg and E.Witten, Nucl.Phys., B426 (1994) 19, hep-th/9407087.

[3] N.Seiberg and E.Witten, Nucl.Phys., B431 (1994) 484, hep-th/9408099.

[4] A.Klemm, W.Lerche, S.Theisen and S.Yankielowicz, Phys. Lett. 344B (1995) 169; hep-th/ 9411048; hepth/ 9412158;

P.Argyres and A.Faraggi, Phys. Rev. Lett. 73 (1995) 3931, hep-th/ 9411057.

[5] M.Toda, Theory of Nonlinear Lattices, Springer-Verlag, 1981.

[6] E.Martinec and N.Warner, Nucl.Phys., 459 (1996) 97-112, hep-th/9509161

[7] O.Bogoyavlensky, Toppling Solitons. Nonlinear Integrable Equations, Moscow, Nauka, 1981.

[8] R.Donagi and E.Witten, Nucl.Phys., B460 (1996) 299, hep-th/9510101;

E.Martinec, Phys.Lett., B367 (1996) 91, hep-th/9510204;

A.Gorsky and A.Marshakov, Phys.Lett., B375 (1996) 127, hep-th/9510224;

H.Itoyama and A.Morozov, Nucl.Phys., B477 (1996) 855, hep-th/9511125; Nucl.Phys., B491 (1997) 529, hep-th/9512161:

E.D'Hoker and D.Phong, hep-th/9709053, hep-th/ 9804126.

[9] N.Nekrasov, Nucl.Phys., B531 (1998) 323, hepth/9609219.

[10] H.Braden, A.Marshakov, A.Mironov and A.Morozov, hepth/9812078; to appear in Phys.Lett. B.

[11] H.Braden, A.Marshakov, A.Mironov and A.Morozov, hep-th/9902205.

[12] B.Dubrovin, I.Krichever and S.Novikov, Integrable systems - I, Sovremennye problemy matematiki (VINITI), Dynamical systems - 4 (1985) 179.

[13] N.Hitchin, Duke. Math. Journ. 54 (1987) 91.

[14] A.Gorsky and N.Nekrasov, hep-th/ 9401021.

[15] E.Witten, hep-th/ 9703166.

[16] D.-E.Diaconescu, hep-th/9608163.

[17] A.Marshakov, M.Martellini and A.Morozov, Phys. Lett. B418 (1998) 294; hep-th/ 9706050.

[18] N.Seiberg and E.Witten, hep-th/9607163. 
[19] E.Date and S.Tanaka, Prog. Theor. Phys. 55 (1976) 457; Prog. Theor. Phys. Suppl. 59 (1976) 107.

[20] I.Krichever, Soviet. Math. Surveys 33 (1978) N4 215; in Appendix to B.Dubrovin, Soviet. Math. Surveys 36 (1981) N2, 12.

[21] K.Ueno and K.Takasaki, Adv.Studies in Pure Math. 4 (1984) 1.

[22] I.Krichever, Func. An. \& Apps. 11 (1977) 15; Soviet. Math. Surveys 32 (1977) 180.

[23] L.Faddeev and L.Takhtadjan, Hamiltonian Approach to the Theory of Solitons, Moscow, Nauka, 1986.

[24] A. Marshakov, in New Developments in Quantum Field Theory, Plenum Press 1999, NATO ASI Series B: Physics 366 (Eds. P.H.Damgaard and J.Jurkiewicz), 279; hep-th/ 9709001.

[25] F.Calogero, J.Math.Phys., 10 (1969) 2191, 2197; 12 (1971) 419; Lett.Nuovo Cimento (2), 13 (1975) 411; 16 (1976) 77;

B.Sutherland, Phys.Rev., A5 (1972) 1372;

J.Moser, Adv.Math., 16 (1975) 1;

M.Olshanetsky and A.Perelomov, Lett.Math.Phys., 2 (1977) 7.

[26] I.Krichever, Func.Anal. \& Appl., 14 (1980) 282.

[27] S.Ruijsenaars and H.Schneider, Ann.Phys.(N.Y.), 170 (1986) 370.

[28] S.Ruijsenaars, Comm.Math.Phys., 110 (1987) 191.

[29] V.Inozemtsev, Comm.Math.Phys., 121 (1989) 629.

[30] S.Ruijsenaars, Comm.Math.Phys., 133 (1990) 217.

[31] A.Hanany and Y.Oz, Nucl. Phys. B452 (1995) 283, hep-th/ 9505075.

[32] A.Marshakov, Mod. Phys. Lett. A11 (1996) 1169; hep-th/ 9602005.

[33] C.Ahn and S.Nam, Phys. Lett. B387 (1996) 304, hep-th/ 9603028.

[34] I.Krichever and D.Phong, J. Diff. Geom. 45 (1997) 349, hep-th/ 9604199.

[35] A.Gorsky, A.Marshakov, A.Mironov and A.Morozov, Phys. Lett. B380 (1996) 75, hep-th/ 9603140.

[36] A.Marshakov, in Proceedings of 10th International Conference "Problems of Quantum Field Theory", Dubna 1996, hep-th/ 9607159.

[37] A.Gorsky, A.Marshakov, A.Mironov and A.Morozov, in Problems in Modern Theoretical Physics, Dubna 1996, 44, hep-th/ 9604078.

[38] E.Sklyanin, J. Sov. Math. 47 (1989) 2473; Func.Anal \& Apps. 16 (1982) 27; 17 (1983) 34.

[39] A.Marshakov and A.Mironov, Nucl. Phys. B518 (1998) 59, hep-th/ 9711156.

[40] A.Bordner, E.Corrigan and R.Sasaki, hep-th/9805106:

A.Bordner, R.Sasaki and K.Takasaki, hep-th/9809068;

A.Bordner and R.Sasaki, hep-th/9812232.

[41] E.D'Hoker and D.Phong, hep-th/9804124; hep-th/9804125; hep-th/ 9903002. 